\title{
Scrub Typhus in Continental Chile, 2016-20181
}

\author{
Thomas Weitzel, \\ Constanza Martínez-Valdebenito, \\ Gerardo Acosta-Jamett, Ju Jiang, \\ Allen L. Richards, Katia Abarca
}

Endemic scrub typhus was recently detected on Chiloé Island in southern Chile. We report a series of cases, acquired over a wide geographical range in continental Chile during 2016-2018, demonstrating that this emerging rickettsial infection is also found on the mainland of South America.

Ccrub typhus is a vectorborne zoonosis caused by OriSentia spp. bacteria; infection carries a potentially severe outcome (1). Although widely underrecognized, scrub typhus is considered one of the most important rickettsial infections worldwide in terms of prevalence and severity (2). Until recently, scrub typhus was associated with only a single species, O. tsutsugamushi, which is transmitted by larvae of trombiculid mites (chiggers) and threatens $>1$ billion human inhabitants within the so-called tsutsugamushi triangle in the Asia-Pacific region (1). Since 2006, the discoveries of scrub typhus in 4 patients on Chiloé Island in Chile $(3,4)$ and in 1 patient from Dubai, United Arab Emirates (5), have suggested the emergence of the disease farther afield (6). This change of paradigm has been reinforced by recent studies mainly from Africa (7). The emergence of endemic scrub typhus on Chiloé Island has been confirmed by ongoing studies of our working group in Chile $(8,9)$. Whether this disease is only endemic to Chiloé Island or has a wider distribution is unknown. We report 9 patients who had scrub typhus diagnosed after visiting different regions of continental Chile during 2016-2018.

\section{The Study}

After the confirmation of autochthonous scrub typhus cases in Chiloé in 2016 (4), the Chilean Ministry of Health issued a clinical alert advising healthcare providers in Chile to confirm possible cases in cooperation with our research group. Most of the patients described in this report were

Author affiliations: Clinica Alemana, Universidad del Desarrollo,

Santiago, Chile (T. Weitzel); Pontificia Universidad Católica de Chile,

Santiago (C. Martínez-Valdebenito, K. Abarca); Universidad Austral

de Chile, Valdivia, Chile (G. Acosta-Jamett); Naval Medical Research

Center, Silver Spring, Maryland, USA (J. Jiang, A.L. Richards) identified as a result of the clinical alert and are included in an ongoing clinical-epidemiologic scrub typhus project.

We tested serum samples obtained during acute and convalescent phases of infection for Orientia-specific antibodies by using a commercial IgG indirect immunofluorescence assay (Fuller Laboratories, http://www.fullerlabs. com), based on whole-cell O. tsutsugamushi Gilliam, Karp, Kato, and Boryong strains, and by using Scrub Typhus Detect IgG and IgM ELISA (InBios International Inc., http:// www.inbios.com) with recombinant $56-\mathrm{kD}$ type-specific antigens of $O$. tsutsugamushi Karp, Kato, Gilliam, and TA716 strains. We examined DNA extracted from eschar material by using 16S rRNA (rrs) and $47 \mathrm{kDa}$ gene ( $h$ trA) seminested PCR and sequencing, as previously described (10), except that we changed the forward primer of the PCR step of rrs from 16SU17 to 16SOR155f. We also tested all samples by using a recently developed Orientia genus-specific quantitative real-time PCR assay targeting $\operatorname{rrs}$ (11).

Among the 37 patients with suspected scrub typhus who were tested during 2016-2018, 13 were from mainland Chile. Of those, 9 had scrub typhus diagnosed both serologically and molecularly. None of these 9 patients lived in or traveled to Chiloé Island or other regions with endemic scrub typhus, but all were exposed to natural habitats on mainland Chile (Table 1). Seven patients were male and 2 female; median age was 28 years.

All cases occurred after outdoor activities during the summer months of February and March. We defined probable exposure sites as those locations where patients reported outdoor activities with close contact to natural environments within the 7-20 days before symptom onset. Most infections were acquired in the Los Lagos Region, which includes Chiloé Island. However, 2 cases were acquired farther south, in the Aysén Region, and another case farther north, in the Bio Bío Region (Figure). The sites of exposure ranged over a total distance of $>1,120$ $\mathrm{km} \quad$ (https://ec.europa.eu/programmes/erasmus-plus/resources/distance-calculator_en), from $38^{\circ} 03^{\prime} \mathrm{S}$ to $47^{\circ} 47^{\prime} \mathrm{S}$. Details of the activities leading to the exposure have been described elsewhere (12).

All patients had fever, generalized maculopapular rash, eschar, and headache; other frequent symptoms were

${ }^{1}$ Preliminary results from this study were presented at the 67th Annual Meeting of the American Society of Tropical Medicine and Hygiene, October 28-November 2, 2018, New Orleans, Louisiana, USA. 
Table 1. Demographic and epidemiologic data of 9 scrub typhus cases in continental Chile, 2016-2018

\begin{tabular}{lcccc}
\hline & & & \multicolumn{2}{c}{ Location of probable exposure } \\
\cline { 3 - 4 } Case no. & Age, y/sex & Month of probable exposure & Region & Site \\
\hline 1 & $43 / \mathrm{M}$ & $2016 \mathrm{Mar}$ & Aysén & Caleta Tortel \\
2 & $56 / \mathrm{M}$ & $2017 \mathrm{Feb}$ & Los Lagos & Cochamón \\
3 & $25 / \mathrm{M}$ & $2017 \mathrm{Mar}$ & Queulat \\
4 & $69 / \mathrm{M}$ & $2018 \mathrm{Feb}$ & Puelo & Puelo \\
5 & $22 / \mathrm{F}$ & $2018 \mathrm{Feb}$ & Aysén & Tos Lagos \\
6 & $25 / \mathrm{M}$ & $2018 \mathrm{Feb}$ & Los Lagos & Tagua \\
7 & $39 / \mathrm{M}$ & $2018 \mathrm{Feb}$ & Los Lagos & Alto Bío Bío \\
8 & $28 / \mathrm{M}$ & $2018 \mathrm{Mar}$ & Bío Bío & Cochamó \\
9 & $21 / \mathrm{F}$ & $2018 \mathrm{Mar}$ & Los Lagos & \\
\hline
\end{tabular}

myalgia (8 patients) and regional lymphadenopathy (5 patients). Laboratory abnormalities included elevated C-reactive protein ( 8 patients) and transaminases (6 patients), thrombocytopenia (4 patients), and leukopenia (4 patients). Eight patients required hospitalization. We noted a serologic response to $O$. tsutsugamushi antigens in all 9 patients. Eight patients were positive for IgG by indirect immunofluorescence assay, mostly with low titers, and 3 of the 6 patients for whom convalescent-phase samples were available showed seroconversion or $\mathrm{a} \geq 4$-fold rise in titer. IgG and IgM results by ELISA were positive in 6 of 9 patients on the basis of local cutoff values (Table 2). All cases were confirmed by detection of Orientia-specific DNA from eschar material using 3 different PCR assays (Table 2). After treatment with doxycycline ( 7 patients) or azithromycin (1 patient), patients recovered rapidly; 1 patient recuperated after 6 days of fever without receiving any scrub typhus-specific treatment.

\section{Conclusions}

Our understanding of the global epidemiology of Orientia spp. as human pathogens has undergone important changes. Most importantly, the paradigm of the geographic limitation of scrub typhus to the Asia-Pacific region, which had been unchallenged since the first scientific description of this disease at the beginning of the last century, has been superseded in light of the recent identification of scrub typhus cases on the Arabian Peninsula (Dubai) and South America (Chiloé Island in Chile) $(4,5)$. The Dubai patient was infected by a distinct Orientia species, Candidatus O. chuto; the complete description of the isolates from Chile is pending.

Further studies in rodents and vectors have demonstrated molecular evidence of Orientia spp. or Orientialike organisms in South Africa, Kenya, Senegal, and France $(6,13,14)$. In addition, serologic reports point to possible human exposure to Orientia microorganisms in Djibouti, Kenya, Republic of the Congo, Cameroon, and Peru $(6,7,15)$. Current data suggest that Candidatus O. chuto might be the Orientia species from Africa and the Arabian Peninsula $(5,14)$. Clinical data from these regions are scarce, but 1 molecularly proven case and 2 cases diagnosed on the basis of serologic results occurred in patients with typical symptoms of scrub typhus $(5,6)$.
The case series we report provides important epidemiologic information for South America, highlighting that scrub typhus is not limited to Chiloé Island but also occurs over a wide range of continental Chile. The actual incidence of the infection remains unknown; the observed increase from 1 case in 2016 to 6 cases in 2018 most probably reflects the growing awareness of scrub typhus among

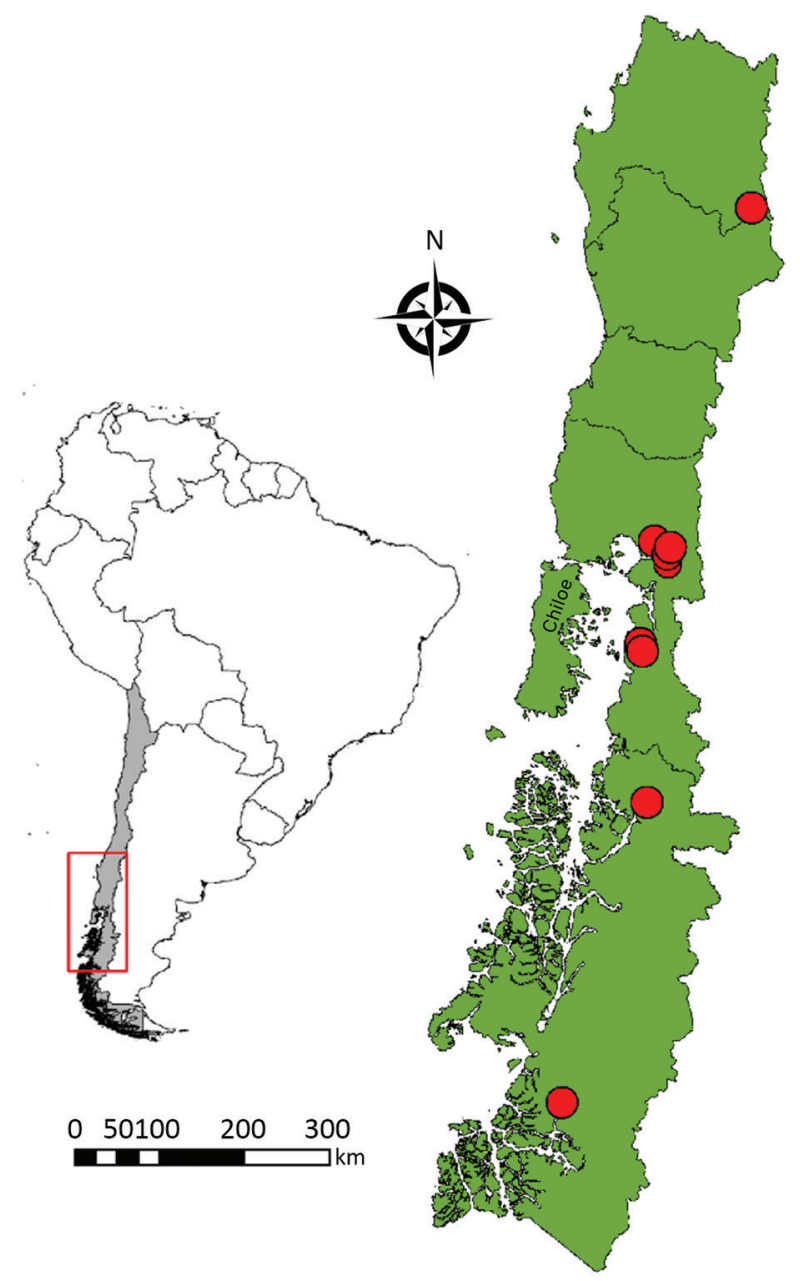

Figure. Locations of probable exposures (red circles) of 9 scrub typhus patients in continental Chile, 2016-2018. Inset map shows the study area (red box) and the location of Chile (gray shading) within South America. 
Table 2. Serologic and molecular diagnosis of scrub typhus cases in continental Chile, 2016-2018*

\begin{tabular}{|c|c|c|c|c|c|c|c|c|c|}
\hline \multirow[b]{3}{*}{ Case no. } & \multicolumn{6}{|c|}{ Serologic testing results } & \multirow{2}{*}{\multicolumn{3}{|c|}{ Eschar molecular testing results }} \\
\hline & \multicolumn{2}{|c|}{ IFA IgG† } & \multicolumn{2}{|c|}{ ELISA IgG $\ddagger$} & \multicolumn{2}{|c|}{ ELISA IgM } & & & \\
\hline & Acute & Conv & Acute & Conv & Acute & Conv & PCR rrs & PCR 47kDa & qPCR rrs \\
\hline 1 & 256 & 256 & + & + & + & - & + & + & + \\
\hline 2 & $\leq 32$ & 128 & - & - & - & - & + & + & + \\
\hline 3 & 64 & 512 & - & + & + & - & + & + & + \\
\hline 4 & 128 & 256 & - & - & - & - & + & + & + \\
\hline 5 & 128 & NA & + & NA & + & NA & + & + & + \\
\hline 6 & 128 & NA & - & NA & - & NA & + & + & + \\
\hline 7 & 128 & 256 & - & + & + & + & + & + & + \\
\hline 8 & $\leq 32$ & NA & + & NA & + & NA & + & + & + \\
\hline 9 & $\overline{6} 4$ & 256 & - & + & - & + & + & + & + \\
\hline
\end{tabular}

infectious diseases physicians in Chile. Because all of the case-patients we describe were included through a surveillance based on passive case detection, we believe that they only represent the tip of the iceberg for scrub typhus in Chile and South America.

The clinical data of this series showed the classical scrub typhus manifestation: fever, generalized maculopapular rash, and eschar at the inoculation site. However, this finding has to be interpreted cautiously because our epidemiologic alert describes these manifestations as typical, which biases the physicians' attention toward these presentations. Less typical clinical signs and symptoms (e.g., without rash or with predominant respiratory symptoms), which are present in Asia in a relevant percentage of cases (1), might go undiagnosed in Chile.

All patients were serologically positive according to $\geq 1$ of the applied commercial assays, which were based on $O$. tsutsugamushi antigens. The low seroreactivity against these antigens, however, suggests a distinct strain or species, which is supported by our preliminary molecular analyses (data not shown). Eschar material, which can be stored and transported under simple conditions (i.e., in a dry plastic tube at $4^{\circ} \mathrm{C}$ ), proved to be the best and most practical specimen, permitting a rapid and reliable confirmation by molecular methods. Studies are ongoing to culture the Orientia species in Chile and to understand its life cycle, including vectors and possible zoonotic reservoirs.

\section{Acknowledgments}

We thank the following physicians, who clinically suspected scrub typhus and permitted the confirmation of the patients of this case series, for their important contribution: Teresa Bidart, Johanna Cabrera, Mario Calvo, María Pilar Gambra, Iván Jara, Miguel Lagos, Alejandra Marcotti, José Manuel Munita, and Luis Thompson.

This work was supported by the Fondo Nacional de Desarrollo Científico y Tecnológico (FONDECYT grant no. 1170810) and the Global Emerging Infectious Diseases Section (work unit no. A1402).
The views expressed in this article are those of the authors and do not necessarily reflect the official policy or position of the Department of the Navy, Department of Defense nor the U.S. Government. A.L.R. is an employee of the US Government. This work was prepared as a part of his official duties. Title 17 U.S.C. $\$ 105$ provides that "copyright protection under this title is not available for any work of the United States Government.” Title 17 U.S.C. \$101 defines a US Government work as a work prepared by a military service member or employee of the US Government as part of that person's official duties. J.J. and A.L.R. provided training and consultation, but did not have contact with patients or participate in the data collection at Naval Medical Research Center, USA.

\section{About the Author}

Dr. Weitzel is a clinical microbiologist and infectious diseases physician specializing in tropical and travel medicine at Clínica Alemana and a professor at the Clínica Alemana Medical School of the Universidad del Desarrollo in Santiago, Chile. His main research interests include vectorborne zoonoses and imported tropical infections.

\section{References}

1. Kim IS, Walker DH. Scrub Typhus. In: Guerrant RL, Walker DH, Weller PF, editors. Tropical infectious diseases: principles, pathogens and practice. 3rd edition. New York: Elsevier Inc.; 2011. p. $334-8$.

2. Paris DH, Shelite TR, Day NP, Walker DH. Unresolved problems related to scrub typhus: a seriously neglected life-threatening disease. Am J Trop Med Hyg. 2013;89:301-7. http://dx.doi.org/ 10.4269/ajtmh.13-0064

3. Balcells ME, Rabagliati R, García P, Poggi H, Oddó D, Concha M, et al. Endemic scrub typhus-like illness, Chile. Emerg Infect Dis. 2011;17:1659-63. http://dx.doi.org/10.3201/eid1709.100960

4. Weitzel T, Dittrich S, López J, Phuklia W, Martinez-Valdebenito C, Velásquez K, et al. Endemic scrub typhus in South America. N Engl J Med. 2016;375:954-61. http://dx.doi.org/10.1056/ NEJMoa1603657

5. Izzard L, Fuller A, Blacksell SD, Paris DH, Richards AL, Aukkanit N, et al. Isolation of a novel Orientia species (O. chuto sp. nov.) from a patient infected in Dubai. J Clin Microbiol. 2010;48:4404-9. http://dx.doi.org/10.1128/JCM.01526-10 
6. Luce-Fedrow A, Lehman ML, Kelly DJ, Mullins K, Maina AN, Stewart RL, et al. A review of scrub typhus (Orientia tsutsugamushi and related organisms): then, now, and tomorrow. Trop Med Infect Dis. 2018;3:8. http://dx.doi.org/10.3390/ tropicalmed3010008

7. Xu G, Walker DH, Jupiter D, Melby PC, Arcari CM. A review of the global epidemiology of scrub typhus. PLoS Negl Trop Dis. 2017;11:e006062. http://dx.doi.org/10.1371/journal.pntd.0006062

8. Weitzel T, Jiang J, Acosta-Jamett G, Martínez-Valdebenito C, López J, Richards AL, et al. Canine seroprevalence to Orientia species in southern Chile: a cross-sectional survey on the Chiloé Island. PLoS One. 2018;13:e0200362. http://dx.doi.org/10.1371/ journal.pone. 0200362

9. Abarca K, Weitzel T, Martínez-Valdebenito C, Acosta-Jamett G. Scrub typhus, an emerging infectious disease in Chile [in Spanish]. Rev Chilena Infectol. 2018;35:696-9.

10. Weitzel T, Aylwin M, Martínez-Valdebenito C, Jiang J, Munita JM, Thompson L, et al. Imported scrub typhus: first case in South America and review of the literature. Trop Dis Travel Med Vaccines. 2018;4:10. http://dx.doi.org/10.1186/s40794018-0070-8

11. Jiang J, Martínez-Valdebenito C, Weitzel T, Abarca K, Richards AL. Development of an Orientia genus-specific quantitative real-time PCR assay and the detection of Orientia species in DNA preparations from O. tsutsugamushi, Candidatus Orientia chuto, and Orientia species from Chile. In: Abstracts of the 29th Meeting of the American Society for Rickettsiology; Milwaukee, USA; 2018 Jun 16-19, Abstract no. 46.
12. Weitzel T, Acosta-Jamett G, Martínez-Valdebenito C, Richards AL, Grobusch MP, Abarca K. Martínez-Valdebenito, Richards AL, Grobusch MP, Abarca K. Scrub typhus risk in travelers to southern Chile. Travel Med Infect Dis. 2019 Jan 9 [Epub ahead of print]. http://dx.doi.org/10.1016/j.tmaid.2019.01.004

13. Kolo AO, Sibeko-Matjila KP, Maina AN, Richards AL, Knobel DL, Matjila PT. Molecular detection of zoonotic rickettsiae and Anaplasma spp. in domestic dogs and their ectoparasites in Bushbuckridge, South Africa. Vector Borne Zoonotic Dis. 2016;16:245-52. http://dx.doi.org/10.1089/vbz.2015.1849

14. Masakhwe C, Linsuwanon P, Kimita G, Mutai B, Leepitakrat S, Yalwala S, et al. Identification and characterization of Orientia chuto in trombiculid chigger mites collected from wild rodents in Kenya. J Clin Microbiol. 2018;56:e1124-18. http://dx.doi.org/ 10.1128/JCM.01124-18

15. Kocher C, Jiang J, Morrison AC, Castillo R, Leguia M, Loyola S, et al. Serologic evidence of scrub typhus in the Peruvian Amazon. Emerg Infect Dis. 2017;23:1389-91. http://dx.doi.org/10.3201/ eid2308.170050

Address for correspondence: Thomas Weitzel, Laboratorio Clínico, Clínica Alemana, Av Vitacura 5951, Santiago, Chile; email: thomas.weitzel@gmail.com; Katia Abarca, Departamento de Enfermedades Infecciosas e Inmunología Pediátricas, Pontificia Universidad Católica de Chile, Diagonal Paraguay 362, Santiago, Chile; email: katia@med.puc.cl

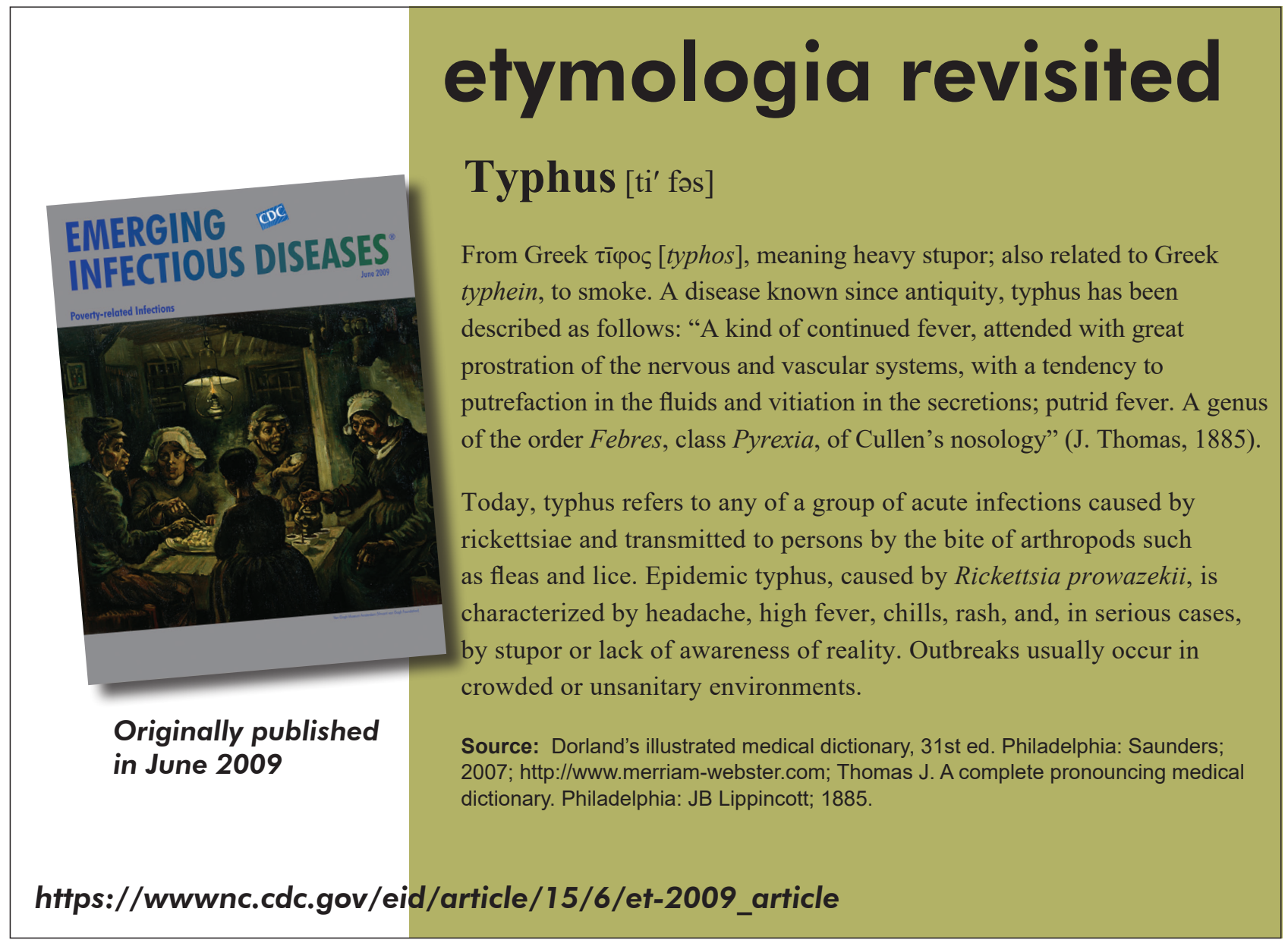

